\title{
Optimal DG location and sizing for minimum active power loss in radial distribution system using firefly algorithm
}

\author{
S. Remha, S. Chettih, S. Arif \\ Amar Telidji University, LACoSERE Laboratory, University of Laghouat, Algeria \\ s.remha@lagh-univ.dz
}

\begin{abstract}
In this paper, a novel optimization algorithm is presented for the optimal location and sizing of Distributed Generation (DG) units on distribution systems. For this purpose, a recently based meta-heuristic called Firefly Algorithm (FA) has been employed to minimize the total active power losses. The results show considerably improved in voltage profiles of all the buses and enhance the voltage stability index. The investigations were tested on IEEE 33 bus radial distribution system. Simulation results demonstrate the effectiveness of firefly algorithm. Comparison with another method is also given.
\end{abstract}

Keywords: Generation, Firefly Algorithm, Active Power Losses, Radial Distribution Network

Received: 18/02/2017 - Accepted: 25/05/2017

\section{Introduction}

Distributed or dispersed generation (DG) is used in order to produce electrical power for a small-scale $(1 \mathrm{~kW}$ to $50 \mathrm{MW}$ ) that is usually connected to or embedded in the distribution system [1]. The advantage of DG are numerous $[2,4]$ and the reasons for implementing DG units are an energy efficiency or rational use of energy, diversification of energy resources, reduction of on-peak operating cost, deferral of network upgrades, minimizing power losses, lower transmission and distribution costs, and possible increase of service quality to the end-customer. Moreover, DGs are available in modular units, characterized by ease of finding sites for smaller generators, shorter construction times, lower capital costs and reduction of gaseous emissions.

In literature different analytical and meta-heuristic optimization based algorithms have been proposed for optimum DG location and sizing. In [5], the authors in [6] have employed an analytical approach for the optimum DG unit location with fitness function of minimization of active power losses. Further, the authors in [7] have developed a new analytical method for the voltage profile enhancement and compensation of active power losses. The authors in [8] have proposed a meta-heuristic technique called Ant Bee Colony (ABT) algorithm for reducing the active power losses in distribution power system. Also, the authors in [9] and [10] have applied a Cuckoo Search Algorithm (CSA) for DG placement and sizing problem in order to reduce the active power loss and to enhance the network voltage profile. In [11], the authors have proposed particle swarm optimization (PSO) algorithm for reducing the power losses in the distribution system. Furthermore, the authors in [12] have proposed on the installation of the capacitor bank with objective function of minimization of total power losses and net saving maximization using a novel optimization algorithm. Other prepositions in [13] based on multiobjective optimization problem for improving the transient stability using a hybrid evolutionary algorithm.

The presents study describes the employment of a novel meta-heuristic called Firefly Algorithm (FA) for the optimal placement and sizing of DG unit in radial distribution networks. This problem is formulated as an optimization problem. The proposed algorithms have been tested on IEEE 33 bus radial distribution systems.

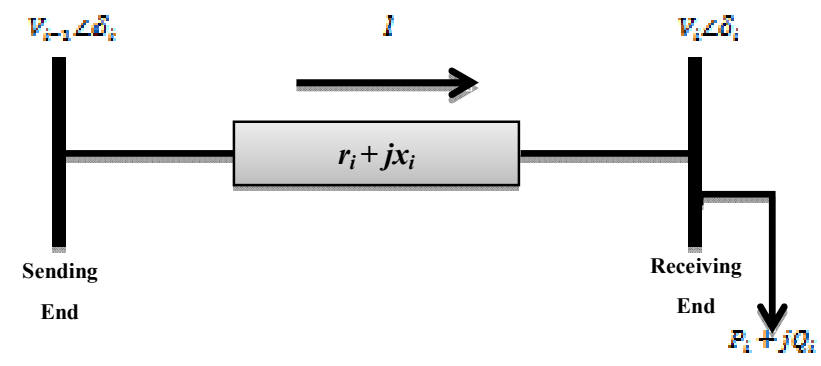

Figure 1. Electrical equivalent of two node system.

This paper is organized as follows: Firstly presents the problem formulation. Section II explains the proposed approach. The results and discussion are presented in Section III and finally the conclusion is given in Section IV 


\section{Problem formulation}

A. Active power loss

In this study, the optimal siting and sizing of DG units in 33 bus radial distribution networks is formulated as an optimization problem to reduce the total active power losses. This latest can be calculated by Eq. 1. Figure 1 shows the electrical equivalent of radial distribution system.

From Figure 1, the following equation can be written:

$$
P L(i)=r_{i} \frac{\left(P_{i}^{2}+Q_{i}^{2}\right)}{V_{i}^{2}}
$$

Therefore, the objective function is calculated using the following equation:

$$
f=\sum_{i=2}^{N_{\text {bus }}} P L=\sum_{i=2}^{N_{\text {bus }}} P L(i)=\sum_{i=2}^{N_{\text {bus }}} r_{i} \frac{\left(P_{i}^{2}+Q_{i}^{2}\right)}{V_{i}^{2}}
$$

Where $i-1$ sending end node; $i$ receiving end node; $r_{i}+j x_{i}$ is the line impedance connected between $i-1$ and $i ; I$ current of branch $j ; V_{i-1}$ voltage of node $i-1 ; V_{i}$ voltage of node $i ; P_{i}$ and $Q_{i}$ active and reactive power load fed through node $i$.

- $\quad$ Total Loss reduction (TLR) [14]:

Total Loss Reduction (TLR) is calculated using Eq. (3)

$$
T L R \%=\frac{\sum_{i=1}^{N_{\text {bus }}} P L_{W / D G}-\sum_{i=1}^{N_{\text {bus }}} P L_{W o / D G}}{\sum_{i=1}^{N_{\text {bus }}} P L_{W o / D G}} \times 100
$$

Where, $P L_{W / D G}$ is the total line losses in the system with the employment of DG and $P L_{W o / D G}$ is the total line losses in the system without DG

\section{Constraints}

The objective function is subjected to main constraints in the optimization process in the proposed methodologies are:

$$
\begin{aligned}
& >\text { Power constraint } \\
& >\text { Voltage constraint. } \\
& >\text { Active power losses constraint. } \\
& >\text { DG size constraint. }
\end{aligned}
$$

\section{B. Power constraint}

The equality constraints are active/reactive power flow equations as:

$$
\left\{\begin{array}{l}
P_{G i}-P_{D i}-V_{i} \cdot \sum_{j=1}^{N_{b u s}} V_{j} \cdot Y_{i j} \cdot \cos \left(\theta_{i j}+\delta_{j}-\delta_{i}\right) \\
Q_{G i}-Q_{D i}-V_{i} \cdot \sum_{j=1}^{N_{b u s}} V_{j} \cdot Y_{i j} \cdot \sin \left(\theta_{i j}+\delta_{j}-\delta_{i}\right)
\end{array}\right.
$$

Where $P_{G i}$ and $Q_{G i}$ are the active (resp. reactive) power generated at the $i$ th bus; $P_{D i}$ and $Q_{D i}$ are the active (resp. reactive) load demand at the same bus; $Y_{i j}$ and $\theta_{i j}$ are the admittance magnitude (resp. angle) of branch connecting bus $i$ and $j$.

C. Voltage constraint

The voltage magnitude must keep within the specified limits at each bus [15]:

$$
V_{\min } \leq V_{i} \leq V_{\max }
$$

Where $V_{\min }, V_{\max }$ are the lower and upper limits of bus voltage, respectively.

D. Active power losses constraint

The losses after installing DG in power grid should be less than or equal losses before installing DG [15].

$$
P L \text { with } D G \leq P L \text { without } D G
$$

\section{E. Distributed generation size constraint}

To obtain a reasonable solution, the size of generator should not be so small or so high with respect to load value. The DG size is considered not less than zero and not more than the total active load demand as following [15]:

$$
0 \leq \text { size of } D G\left(P_{D G}\right) \leq \sum_{i=1}^{N_{\text {bus }}} P_{D i}
$$

Where $\mathrm{P}_{\mathrm{Di}}$ is the active load demand at the same bus.

\section{Proposed algorithm}

Firefly Algorithm (FA) is invented by Xin-She Yang [15] for solving multimodal optimization problem. The development of FA is based on flashing behavior of fireflies. There are about two thousand firefly species where the flashes often unique for a particular species. The flashing light is produced by a process of bioluminescence where the exact functions of such signaling systems are still on debating. Nevertheless, two fundamental functions of such flashes are to attract mating partners (communication) and to attract potential prey.

For simplicity, the following three ideal rules are introduced in FA development [16]:

$>$ all fireflies are unisex so that one firefly will be attracted to other fireflies regardless of their sex,

$>$ attractiveness is proportional to their brightness, thus for any two flashing fireflies, the less brighter one will move towards the brighter one,

$>$ The brightness of a firefly is affected by the landscape of the objective function.

For maximization problem, the brightness can simply be proportional to the value of the objective or fitness function.

\section{Application of the Firefly Algorithm to the problem}

In this section, Firefly Algorithm (FA) is proposed to discover the best placement and sizing of Distributed generation (DG) unit in a radial distribution system to 
minimize the total active power loss in the system. By reducing power loss, the voltage profile at each bus is also expected to be improved the voltage profiles and increased values of voltage stability index. Figure 2 presents the general flowchart of Firefly algorithm for evaluating the objective function.

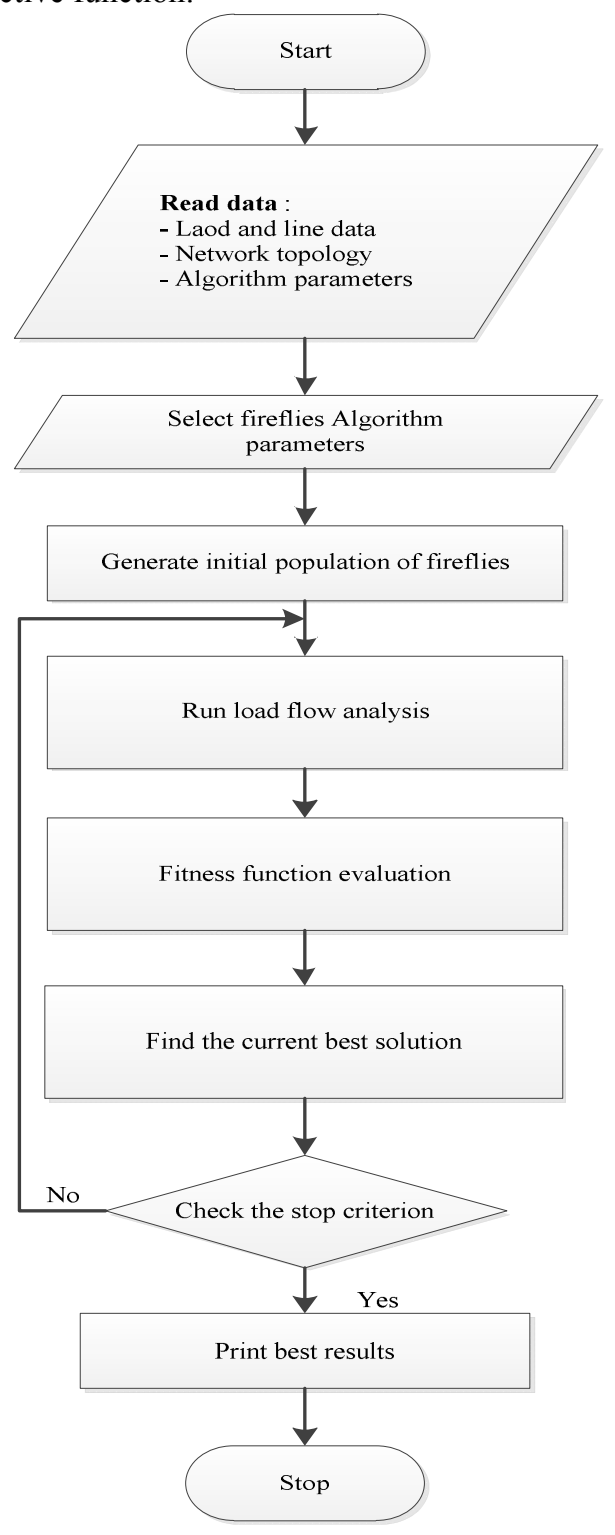

Figure 2. Flowchart of DG location and sizing using Firefly algorithm.

\section{Resultats and discussion}

In this study, the proposed algorithms are used to determine the optimal location and size of the DG unit and they have been carried out on IEEE 33-bus radial distribution system. The FA properties in this simulation are set as follow:
TABLE I. FIREFLY ALGORITHM SETTINGS

\begin{tabular}{||c||c||}
\hline Settings & Values \\
\hline \hline Number of iteration & 50 \\
\hline \hline Number of population & 20 \\
\hline \hline Variation of attractiveness & 0.5 \\
\hline \hline Randomization & 0.8 \\
\hline \hline Absorption coefficient & 1 \\
\hline
\end{tabular}

- IEEE 33-bus Radial Distribution System

The test system is IEEE 33-bus radial distribution system, with the total load is $3.715 \mathrm{MW}$ and 2.3 MVar, its voltage level is $12.66 \mathrm{kV}$, this system contains 33 bus and 32 branches. Its corresponding scheme is presented in Figure 3 [17].

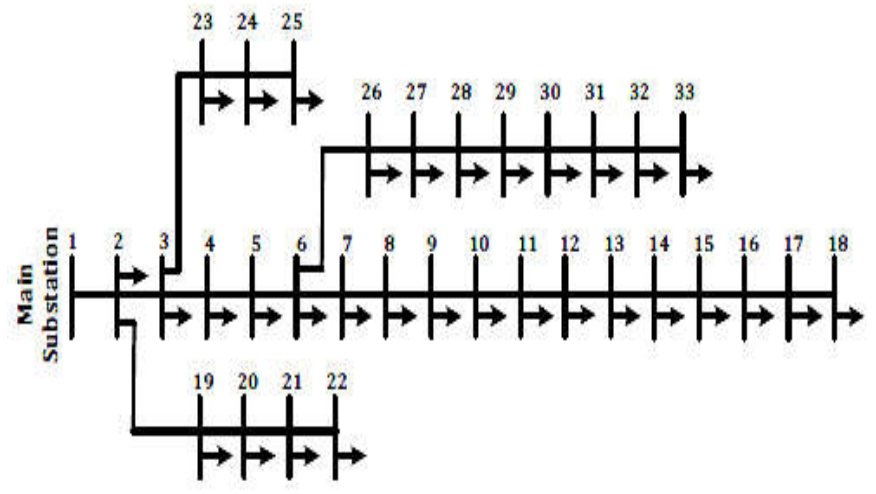

Figure 3. IEEE 33-Bus radial distribution system

F. Results

The DG unit localization, corresponding sizes and active power losses in the cases without and with DG unit using the proposed algorithm and PSO algorithm find by [11] are respectively summarized in TABLE II. The voltage profile, minimum and maximum voltage, voltage stability index, minimum and maximum voltage stability index and the active power losses are presented in Figures. (4-8) simultaneously.

TABLE II. APPLICATION OF PROPOSED ALGORITHM ON IEEE 33 TEST SYSTEM

\begin{tabular}{cccc}
\hline \hline \multicolumn{4}{c}{ IEEE 33-Bus Test System } \\
\hline \hline & Without DG & PSO [11] & $\begin{array}{c}\text { Proposed } \\
\text { Method }\end{array}$ \\
\hline \hline Optimum location & - & 7 & 6 \\
Optimum DG size (MWA) & - & 2.91 & 2.60 \\
Active Power Losses (KW) & 211,00 & 115.1 & 111 \\
Loss reduction \% & - & 45.45 & 47.39 \\
\hline \hline
\end{tabular}

\section{G. Discussion}

The effect of DG placement is also measured by calculating the active power loss, the voltage profiles and 
the voltage stability index. Table III shows the effect of DG placement on system performance. From these results, we can observe the advantage of DG units in improvement of system performances, as reduction of total system losses and the voltage index stability enhancement. In order to validate the performance of the proposed algorithm in this study, we have used IEEE 33-bus test distribution system.

When the proposed method is applied on test system, it was found that the proposed method gives much better voltage profile as compared to the base case. Fig. 4 shows voltage profile of IEEE 33-bus test distribution system (with and without DG placement). From Fig. 5, it can be observed that the minimum voltage is raised from $0.90 \mathrm{pu}$ in the case without DG unit to $0.94 \mathrm{pu}$ in the case with DG unit using the proposed method.

Fig. 6 shows the voltage stability indices of test system. The weakness of voltage stability indices for all buses in the distribution system before integration DG unit is obvious. But after integration DG unit, the buses stability indexes are considerably enhanced. Fig. 7 shows the minimum and maximum voltage stability index of the test system (with and without DG placement).

The proposed method also results in total power loss reduction. TABLE II shows the effect of DG placement on loss reduction, using the proposed and another existing method. From TABLE II, it can be observed after installation of DG units, the active power losses have been significantly reduced. The proposed algorithm is found better in performance than PSO algorithm. Where the proposed algorithm gives $2 \%$ more power loss than PSO algorithm. Fig. 8 shows the active power loss at buses with and without DG for IEEE 33 bus test system.

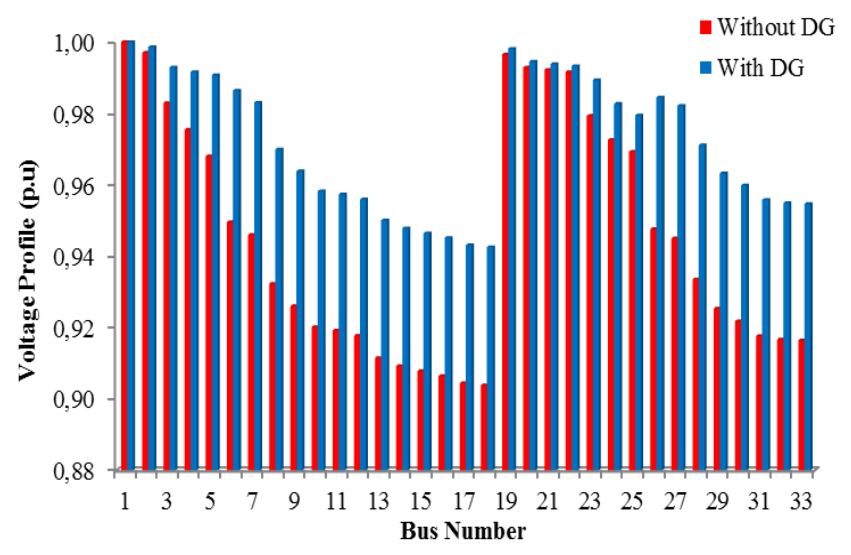

Figure 4. Voltage profiles of 33 bus system.

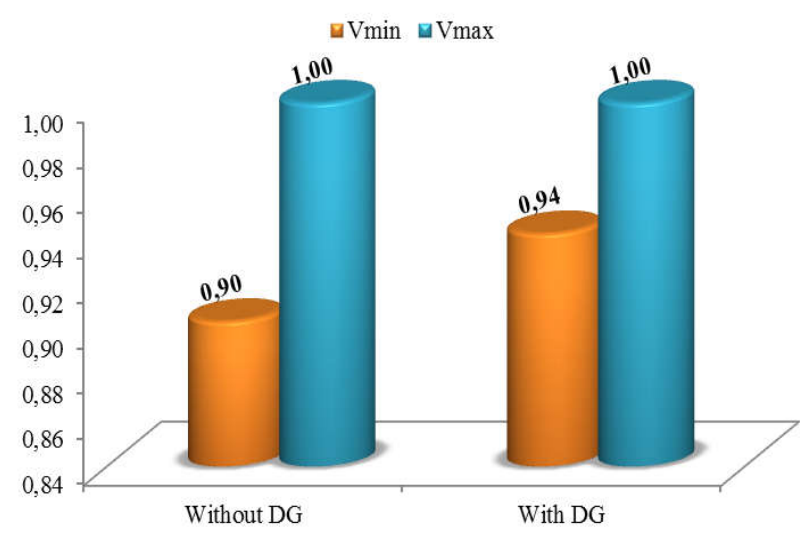

Figure 5. Minimum and Maximum voltage of 33 bus system.

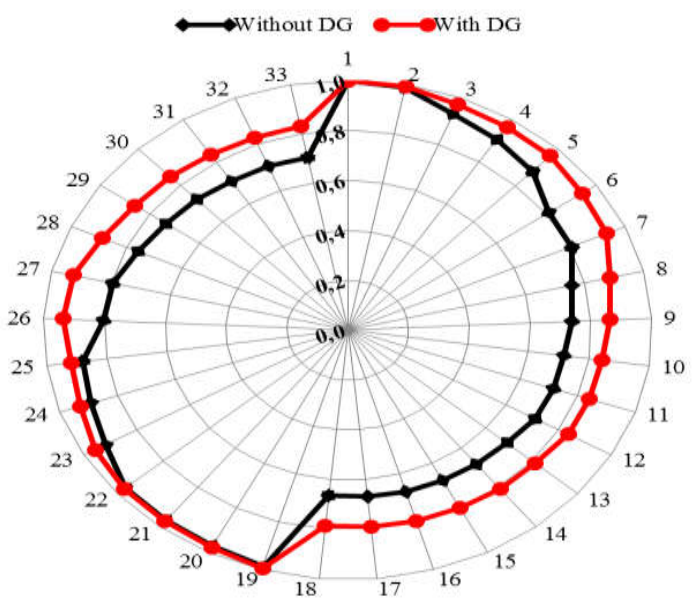

Figure 6. Voltage stability index of 33 bus system

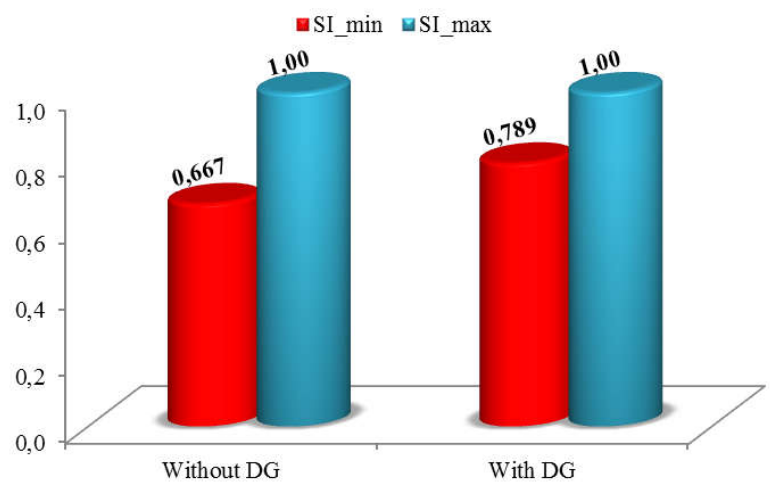

Figure 7. Minimum and Maximum voltage stability index of 33 bus system 


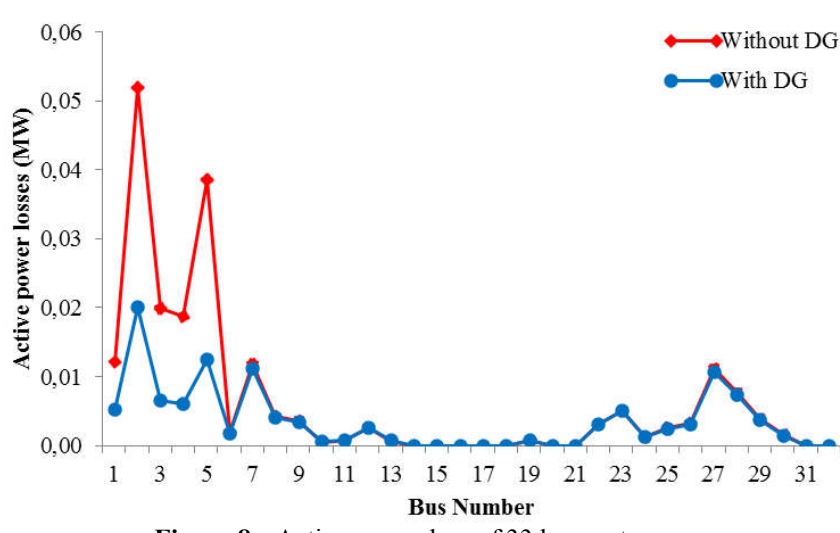

Figure 8. Active power loss of 33 bus system.

\section{Conclusion}

In this paper, the problem of optimal DG unit placement and sizing in radial distributed network has been treated. For this reason, a recent Meta-heuristic technic known as firefly Algorithm is employed to minimize the total active power losses. Its effectiveness and robustness is compared to the particle swarm optimization algorithm. Simulations were performed on IEEE 33 bus radial system. The obtained results clearly reveal the effectiveness of the proposed algorithm in total active power losses minimizing compared to PSO algorithm.

\section{References}

[1] R. D. Mohammedi et al, Optimal DG Placement and Sizing in Radial Distribution Systems Using NSGA-II for Power Loss Minimization and Voltage Stability Enhancement, International Review of Electrical Engineering, vol 8, Nov/Dec 2013.

[2] P.A. Daly, J. Morrison, Understanding the potential benefits of distributed generation on power delivery systems, Rural Electri Power Conference, 29 April - 1 May 2001, pp. A211 - A213.

[3] P. Chiradeja, R. Ramakumar, An approach to quantify the technical benefits of distributed generation, IEEE Trans Energy Conversion, vol. 19 , no. 4, 2004, pp. 764-773.

[4] M. Maamir, A. Betka, H. Aboub, Modeling and Simulation of Energy Management Hybrid Sources System composed of Solar-PV and Battery, International Journal of Energetica, vol. 1, no. 1, 2016, pp. 12-19.
[5] Caisheng W, Nehrir MH, Analytical approaches for optimal placement of distributed generation sources in power systems. IEEE T Power Syst 2004;19(4):2068-76.

[6] T. Gözel et al, An Analytical Method for the Sizing and Siting of Distributed Generators in Radial Systems, Electric Power Systems Research, vol. 79, 2009, pp. 912-918.

[7] A.Lakshmi Devi et al, A New Analytical Method for the Sizing and Siting of DG in Radial System to Minimize Real Power Losses, International Journal Of Computational Engineering Research, Vol. 2 Issue. 7, November 2012.

[8] R. S. Rao et al, Optimization of Distribution Network Configuration for Loss Reduction Using Artificial Bee Colony Algorithm, World Academy of Science, Engineering and Technology 45 (2008), pp 708-714.

[9] Z. Moravej et al, A Novel Approach Based on Cuckoo Search for DG Allocation in Distribution Network, International Journal of Electrical Power \& Energy Systems, vol. 44, 2013, pp. 672-679.

[10] W. S. Tan et al, Allocation and Sizing of DG Using Cuckoo Search Algorithm, in Power and Energy (PECon), 2012 IEEE International Conference on, 2012, pp. 133-138.

[11] S. Kansal et al, Optimal placement of distributed generation in distribution networks, International Journal of Engineering, Science and Technology, Vol. 3, No. 3, 2011, pp. 47-55

[12] T. V. Kumar et al, Implementation of Novel Optimization Algorithm for Optimal Placement and Sizing of Capacitor Banks in Radial Distribution Systems for Power Loss Minimization and Net Savings Maximization, International Journal of Computer Applications 108, December 2014.

[13] M. Nayeripour et al, Multi-objective Placement and Sizing of DGs in Distribution Networks Ensuring Transient Stability Using Hybrid Evolutionary Algorithm, Renewable and Sustainable Energy Reviews 25, 2013, pp759-767.

[14] M.M. Aman et al, A new approach for optimum DG placement and sizing based on voltage stability maximization and minimization of power losses, Energy Conversion and Management 70, 2013, pp 202-210.

[15] M. Begovic et al, Summary of System Protection and Voltage Stability, Power Delivery, IEEE Transactions on, vol. 10, 1995, pp 631-638.

[16] X.-S. Yang, Firefly Algorithms for Multimodal Optimization, Stochastic Algorithms: Foundation and Applications SAGA 2009, vol.5792, 2009, pp. 169-178.

[17] M. Jamil et al, Optimal Allocation of SPV Based DG System for Loss Reduction and Voltage Improvement in Radial Distribution Systems using Approximate Reasoning, in Power Electronics (IICPE), 2012 IEEE 5th India International Conference on, 2012, pp. $1-5$. 